\title{
Phase diagram and magnons in quasi-one-dimensional dipolar antiferromagnets
}

\author{
M. Hummel and F. Schwabl \\ Physik-Department, Technische Universität München, D-85747 Garching, Germany \\ C. Pich \\ Physics Department, University of California, Santa Cruz, CA 95064
}

(October 15, 2018)

We investigate antiferromagnetic spin chains, which are coupled by a weak antiferromagnetic exchange interaction. The spins are located on a hexagonal lattice, i.e. frustration is present when three-dimensional order sets in. Typical realizations of such systems are the halides $\mathrm{ABX}_{3}$. In this work we particularly study the role of the long-range dipolar interaction within the framework of a Heisenberg model with nearest-neighbor exchange and additional dipolar interaction. We perform a classical ground-state analysis and show that the spin configuration is sensitively dependent on $\kappa^{\prime}$, the ratio of the dipolar interaction to the interchain interaction, as a consequence of their competing character. Several commensurate and incommensurate phases arise in the different regions of the parameter space. The ground-state investigations are supplemented by a stability analysis by means of a linear spin-wave calculation. From the magnon spectra we can show that all commensurate phases are stable against fluctuations. In comparison with experiments $\left(\mathrm{CsMnBr}_{3}, \mathrm{RbMnBr}_{3}\right)$ we obtain good agreement for the energy gaps. From this we conclude that the dipolar interaction is the most important source of anisotropy in these Mn-compounds.

\section{INTRODUCTION}

Unconventional magnetic systems have attracted the interest of experimental and theoretical physicists in the last few years [1]. In these systems competing interactions and/or geometric frustration due to the underlying lattice can lead to unconventional ground states, magnon spectra and magnetic phase diagrams [2]. Furthermore, fluctuations are enhanced in systems with frustrated ground states as well as in low dimensions.

An interaction, which is often competing with respect to the exchange interaction is the dipole-dipole interaction [3]. In real systems the dipole-dipole interaction (DDI) is always present in addition to the short-range exchange interaction. Although its energy is lower than the exchange energy it plays an important role in lowdimensional systems due to its anisotropic and long-range character.

The most famous quasi-one-dimensional systems are the ternary compounds $\mathrm{ABX}_{3}$ (A alkaline, B transition metal and $\mathrm{X}$ halogen), which have been studied intensively theoretically and experimentally in the context of Haldane's phase [4] and solitonic excitations [5]. In these systems the carrier of the magnetic moment, the B-ions, are located on a hexagonal lattice [6]. In this work, the Mn compounds are of particular interest. Because the angular momentum $L$ is zero, no crystal-field splitting occurs in these systems and the dipole-dipole interaction should be the most important anisotropy.

The influence of the DDI in quasi-one-dimensional, antiferromagnetic spin chain systems has not yet been studied very thoroughly. Instead, the DDI is often replaced by a single-ion anisotropy [7] or the coupling between spin chains is neglected [8].

\section{MODEL}

The Hamiltonian of the dipolar antiferromagnet reads

$$
H=-\sum_{l \neq l^{\prime}} \sum_{\alpha \beta}\left(J_{l l^{\prime}} \delta_{\alpha \beta}+A_{l l^{\prime}}^{\alpha \beta}\right) S_{l}^{\alpha} S_{l^{\prime}}^{\beta},
$$

with spins $\mathbf{S}_{l}$ at hexagonal lattice sites $\mathbf{x}_{l}$. The first term describes the exchange interaction $J_{l l^{\prime}}$ which includes the intrachain as well as the interchain interaction. In the following we consider only nearest-neighbor exchange, i.e.

$$
J_{l l^{\prime}}=\left\{\begin{array}{lll}
-J & l, l^{\prime} & \text { along the chains } \\
-J^{\prime} & l, l^{\prime} & \text { within the basal plane }
\end{array} .\right.
$$

For a hexagonal lattice the Fourier transform of the exchange energy is given by

$$
J_{\mathbf{q}}=-2 J \cos q_{z}-2 J^{\prime}\left(\cos q_{x}+2 \cos \left(\frac{q_{x}}{2}\right) \cos \left(\frac{\sqrt{3}}{2} q_{y}\right)\right) \text {. }
$$

Here and in the following we measure wave vectors in chain direction in units of $1 / c$, and wave vectors within the basal planes in units of $1 / a$. The second term in Eq. (1) is the classical dipole-dipole interaction

$$
\begin{aligned}
A_{l l^{\prime}}^{\alpha \beta}=-\frac{1}{2}\left(g \mu_{B}\right)^{2} & \left(\frac{\delta_{\alpha \beta}}{\left|\mathbf{x}_{l}-\mathbf{x}_{l^{\prime}}\right|^{3}}\right. \\
& \left.-\frac{3\left(\mathbf{x}_{l}-\mathbf{x}_{l^{\prime}}\right)_{\alpha}\left(\mathbf{x}_{l}-\mathbf{x}_{l^{\prime}}\right)_{\beta}}{\left|\mathbf{x}_{l}-\mathbf{x}_{l^{\prime}}\right|^{5}}\right) .
\end{aligned}
$$

This term is evaluated by means of the Ewald summation technique [9, 10, which allows the consideration of the long-range nature of the three-dimensional DDI in terms of fast convergent sums. 


\section{GROUND STATES}

In this section we calculate the classical ground states of the system as a function of the ratio of the dipolar energy to the interchain exchange interaction

$$
\kappa^{\prime}=\frac{\left(g \mu_{B}\right)^{2}}{V_{z} J^{\prime}}
$$

where $V_{z}=\frac{\sqrt{3}}{2} a^{2} c$ is the volume of the primitive cell. Due to the quasi-one-dimensionality of the systems (the ratio $J^{\prime} / J$ is $10^{-2} \ldots 10^{-3}$ ) we consider only antiferromagnetic spin configurations along the chain axis, i.e., we restrict $q_{z}$ to $\pi$. The ground-state energy reads

$$
E_{g}=-\sum_{l \neq l^{\prime}} S_{l} M_{l l^{\prime}} S_{l^{\prime}}=-\sum_{\mathbf{q}} S_{\mathbf{q}} M_{\mathbf{q}} S_{-\mathbf{q}}
$$

with

$$
M_{\mathbf{q}}=\left(\begin{array}{ccc}
J_{\mathbf{q}}+A_{\mathbf{q}}^{11} & A_{\mathbf{q}}^{12} & A_{\mathbf{q}}^{13} \\
A_{\mathbf{q}}^{12} & J_{\mathbf{q}}+A_{\mathbf{q}}^{22} & A_{\mathbf{q}}^{23} \\
A_{\mathbf{q}}^{13} & A_{\mathbf{q}}^{23} & J_{\mathbf{q}}+A_{\mathbf{q}}^{33}
\end{array}\right)
$$

where $A_{\mathbf{q}}^{13}=A_{\mathbf{q}}^{23}=0$ for $q_{z}=\pi$. The ground states are specified by those wave vectors which belong to the largest eigenvalues of the matrix $M_{\mathbf{q}}$ in Eq. (6). We obtain the following phases for decreasing $\kappa^{\prime}$ (for the lattice constants we took the values of $\mathrm{CsMnBr}_{3}$ [11):

1. Ferromagnetic phase: $\kappa^{\prime}>\kappa_{1}^{\prime}=200.50$

In the region where the dipolar energy is large compared to the interchain exchange, the minimum of the ground-state energy is reached at $\mathbf{q}_{1}=(0,0, \pi)$. This means that spins within basal planes are ordered ferromagnetically, but still antiferromagnetically along the chains.

2. Incommensurate phase I: $200.12=\kappa_{2}^{\prime}<\kappa^{\prime}<\kappa_{1}^{\prime}$

The ground state is an incommensurate phase in this parameter region. The wave vector moves continuously from $\mathbf{q}_{1}$ to the wave vector $\mathbf{q}_{2}=$ $\left(0, \frac{2 \pi}{\sqrt{3}}, \pi\right)$ (thick solid line in Fig. 1) or any other path rotated by $60^{\circ}$ (dashed lines in Fig. 1).

3. Collinear phase: $17.25=\kappa_{3}^{\prime}<\kappa^{\prime}<\kappa_{2}^{\prime}$

Spins within basal planes are oriented ferromagnetically in chains, that are aligned antiferromagnetically to one another (see Fig. 2). Because of the six-fold symmetry, there are six such ground states resulting from rotation of the ferromagnetic chains. Note that the continuous degeneracy is lifted.

4. Incommensurate phase II: $0<\kappa^{\prime}<\kappa_{3}^{\prime}$

In this incommensurate phase the wave vector moves from $\mathbf{q}_{2}$ to $\mathbf{q}_{3}=\left(\frac{2 \pi}{3}, \frac{2 \pi}{\sqrt{3}}, \pi\right)$. The incommensurability appears because of the finite slope of the dipolar tensor at $\mathbf{q}_{3} \sqrt{12}$ and the parabolic behavior of the exchange energy.

\section{5. $120^{\circ}$-structure: $\kappa^{\prime}=0$}

This ground state is characterized by a threesublattice spin configuration in each basal plane.

The coupling of the spin space to the real space induced by the DDI forces the spins to align within the lattice basal planes for all four phases, in which the DDI is nonzero. Thus, the DDI leads to an in-plane anisotropy.

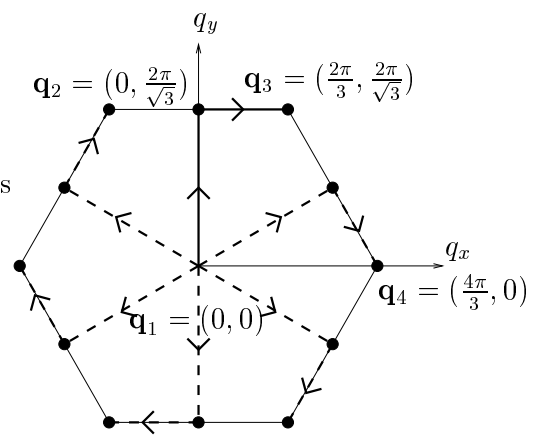

FIG. 1. Paths of the wave vector that minimize the ground-state energy in the Brillouin zone of the hexagonal lattice $\left(q_{z}=\pi\right)$.

The phase diagram for the whole parameter region of $\kappa^{\prime}$ is shown in Fig. 22.

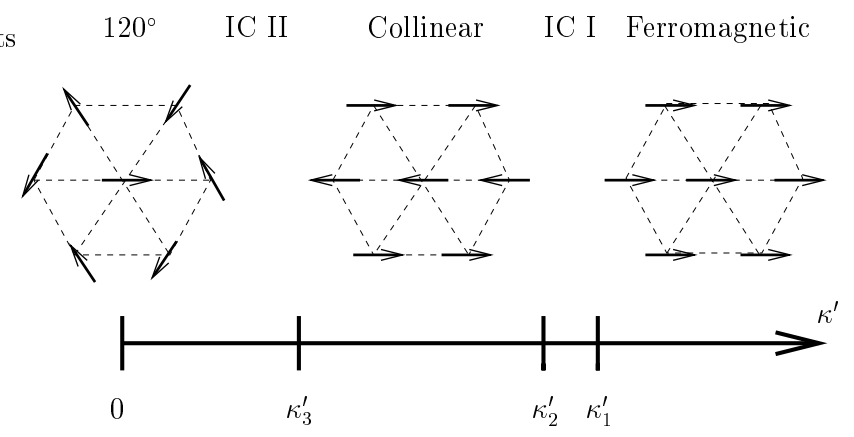

FIG. 2. Spin configurations within the basal plane for different ratios of dipolar to interchain interaction $\kappa^{\prime}$. Only for $\kappa^{\prime}=0$ the $120^{\circ}$-structure is established. For infinitesimal $\kappa^{\prime}$ the phase IC II is favored. The spin configurations of incommensurate phases are not sketched.

In summary, we find three commensurate and two incommensurate phases for arbitrary value of $\kappa^{\prime}$.

\section{MAGNON SPECTRA}

The spin-wave calculation is of interest in its own right and also serves to scrutinize the stability of the phases found against fluctuations. To that end we write the Hamiltonian (11) in terms of creation and annihilation operators employing the Holstein-Primakoff transformation [13]. For low temperatures an expansion up to bilinear terms can be used, leading to linear spin-wave theory. 
The Hamiltonian then is diagonalized with a Bogoliubov transformation from which we obtain the spin-wave frequencies. This investigation is restricted to the commensurate phases, because the infinite primitive cell of the incommensurate phases resists such an analysis.

\section{Ferromagnetic phase}

The magnon spectrum of the ferromagnetic phase is stable for the parameter region given in Sec. III. The rotational invariance of the spins around the chain axis leads to a Goldstone mode in the spectrum [14].

\section{Collinear phase}

The collinear phase of Sec. III is also stable against fluctuations. There is no Goldstone mode due to the discrete degeneracy of the ground state.

\section{$120^{\circ}$-structure}

We argued in Sec. III that the $120^{\circ}$-structure is unstable for infinitesimal dipolar energy due to a linear slope of the dipolar tensor at the ordering wave vector. However, we performed a spin-wave calculation based on a commensurate $120^{\circ}$-structure, where the spins are located within the basal planes of the lattice [15]. It turns out that the spin-wave spectrum is stable for weak dipolar energies, from which we conclude that this commensurate ground state is a good approximation.

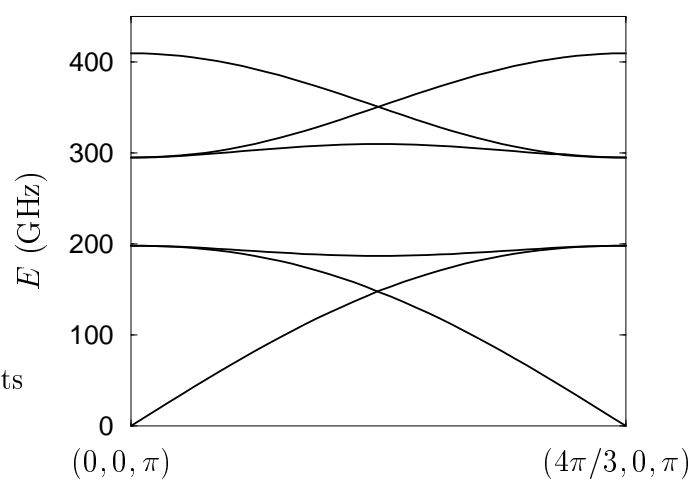

FIG. 3. Magnon spectrum of the $120^{\circ}$-structure perpendicular to the chain direction.

The spin-wave frequencies resulting for the dipolar $120^{\circ}$ structure for $\mathrm{CsMnBr}_{3}$ are shown in Fig. 3, where we used $J=215 \mathrm{GHz}$ and $J^{\prime}=0.41 \mathrm{GHz}$ respectively [16]. This leads to $\kappa^{\prime}=0.774$, i.e., this substance is in the IC II region of the phase diagram in Fig. 2. Including the DDI, from the three Goldstone modes only one survives reflecting the unchanged rotational symmetry around the chain axis.

The spin-wave gaps at $\mathbf{q}=0$ amount to $E_{0,1}=$ $198 \mathrm{GHz}, E_{0,2}=295 \mathrm{GHz}$ and $E_{0,3}=410 \mathrm{GHz}$ for $\mathrm{CsMnBr}_{3}$, which compares favorably with the experimental values [16,17]. Note that this calculation has no free parameter to fit, since the dipolar energy is determined by the lattice constants. We also calculated the spinwave gaps for $\mathrm{RbMnBr}_{3}$; neglecting crystal distortions we also obtain good agreement with the experiment [18].
Thus, we do not need any single-ion anisotropy to explain these results.

\section{SUMMARY}

We found three commensurate and two incommensurate phases for different values of the ratio of dipolar to interchain interaction due to the competing character of those two interactions. We showed via linear spin-wave theory that all commensurate phases are stable against fluctuations and that the incommensurate phase IC II can be approximated by a $120^{\circ}$-structure for weak dipolar energies.

The spin-wave gaps of $\mathrm{CsMnBr}_{3}$ and $\mathrm{RbMnBr}_{3}$ are in good agreement with the experiment, which shows that the dipolar energy is the most important source of anisotropy in these Mn-compounds.

This work has been supported by the BMBF under contract number 03-SC5-TUM 0 and the DFG under contract number PI 337/1-2.

[1] A.T. Skjeltorp and D. Sherrington, editors, Dynamical Properties of Unconventional Magnetic Systems, (Kluwer Academic Publishers, Dordrecht, Boston and London, 1998).

[2] H.T. Diep, editor, Magnetic Systems with Competing Interactions, World Scientific, Singapore (1994).

[3] C. Pich and F. Schwabl, Z. Phys. B 104, 165 (1997).

[4] F.D.M. Haldane, Phys. Rev. Lett. 50, 1153 (1983).

[5] M. Steiner, M.J. Mikeska, Adv. Phys. 40, 191 (1991).

[6] M.F. Collins and O.A. Petrenko, Can. J. Phys. 9, 75 (1997).

[7] H. Kadowaki, K. Hirakawa and K. Ubukoshi, J. Phys. Soc. Jap. 52, 1799 (1983).

[8] R.E. Dietz, L.R. Walker, F.S.L. Hsu and W.H. Haemmerle, Sol. St. Comm. 15, 1185 (1974).

[9] P.P. Ewald, Ann. Phys. (Leipz.) 64, 253 (1921).

[10] L. Bonsall and A.A. Maradudin, Phys. Rev. B 15, 1959 (1977).

[11] J. Goodyear and D.J. Kennedy, Acta Cryst. B 28, 1640 (1972).

[12] H. Shiba, N. Suzuki, J. Phys. Soc. Jpn. 51, 3488 (1982).

[13] T. Holstein and H. Primakoff, Phys. Rev. 58, 1098 (1940).

[14] M. Hummel, C. Pich and F. Schwabl, to be published.

[15] J.A. Oyedele and M.F. Collins, Can. J. Phys. 56, 1482 (1978).

[16] U. Falk, A. Furrer, H.U. Güdel and J.K. Kjems, Phys. Rev. B 35, 4888 (1987).

[17] B.D. Gaulin, M.F. Collins and W.J.L. Buyers, J. Appl. Phys. 61, 3409 (1987).

[18] L. Heller, M.F. Collins, Y.S. Yang and B. Collier, Phys. Rev. B 49, 1104 (1994). 